\title{
A Reversible Theory of Entanglement and its Relation to the Second Law
}

\author{
Fernando G.S.L. Brandãd* and Martin B. Plenid \\ Institute for Mathematical Sciences, Imperial College London, London SW7 2BW, UK and \\ QOLS, Blackett Laboratory, Imperial College London, London SW7 2BW, UK
}

\begin{abstract}
We consider the manipulation of multipartite entangled states in the limit of many copies under quantum operations that asymptotically cannot generate entanglement. As announced in [Brandão and Plenio, Nature Physics 4, 8 (2008)], and in stark contrast to the manipulation of entanglement under local operations and classical communication, the entanglement shared by two or more parties can be reversibly interconverted in this setting. The unique entanglement measure is identified as the regularized relative entropy of entanglement, which is shown to be equal to a regularized and smoothed version of the logarithmic robustness of entanglement.

Here we give a rigorous proof of this result, which is fundamentally based on a certain recent extension of quantum Stein's Lemma proved in [Brandão and Plenio, Commun. Math. 295, 791 (2010)], giving the best measurement strategy for discriminating several copies of an entangled state from an arbitrary sequence of non-entangled states, with an optimal distinguishability rate equal to the regularized relative entropy of entanglement. We moreover analyse the connection of our approach to axiomatic formulations of the second law of thermodynamics.
\end{abstract}

\section{INTRODUCTION}

A basic feature of many physical settings is the existence of constraints on physical operations and processes that are available. These constraints generally imply the existence of resources that can be consumed to implement operations that are otherwise forbidden due to the constraints that have been imposed. Examples include an auxiliary heat bath in order to decrease the entropy of a thermodynamical system [1] or prior secret correlations for the establishment of secret key between two parties who can only operate locally and communicate by a public channel [2]. In quantum information theory one often considers the scenario in which two or more distant parties want to exchange quantum information, but are restricted to act locally on their quantum systems and communicate classical bits only. A resource of intrinsic quantum character, entanglement, allows the parties to completely overcome the limitations caused by the locality requirement on the quantum operations available [3].

Resource theories are considered in order to determine when a physical system, or a state thereof, contains a given resource; to characterize the possible conversions from a state to another when one has access only to a restricted class of operations which cannot create the resource for free; and to quantify the amount of such a resource contained in a given system.

One may try to analyse the above questions at the level of individual systems. However, it is natural to expect that a simplified theory will emerge when instead one looks at the bulk properties of a large number of systems. An illustrative example of such a type of theory is thermodynamics, which describes the physics of bulk properties of large systems in equilibrium by a very simple set of rules of universal character. In the context of its second law, in particular,

*Electronic address: fernando.brandao@imperial.ac.uk

${ }^{\dagger}$ Electronic address: m.plenio@imperial.ac.uk 
the theory determines in terms of a unique quantity - the entropy - which transformations from one thermodynamical equilibrium state into another are possible by means of adiabatic processes. There is a long history of examinations of the foundations underlying the second law, starting with Carathéodory work in the beginning of last century [4]. Of particular interest in the present context is the work of Giles [5] and notably Lieb and Yngvason [6, 7], stating that there exists a total ordering of equilibrium thermodynamical states that determines which state transformations are possible by means of an adiabatic process. From simple, abstract, axioms one can show the existence of an entropy function $S$ fully determining the achievable transformations: given two equilibrium states $A$ and $B, A$ can be converted by an adiabatic process into $B$ if, and only if, $S(A) \leq S(B)$.

It was noted early on in the development of entanglement theory that the same total order for state transformations is found in the manipulation of bipartite pure states by local operations and classical communication (LOCC), in the asymptotic limit of an arbitrarily large number of copies of the states. Given two bipartite pure states $\left|\psi_{A B}\right\rangle$ and $\left|\phi_{A B}\right\rangle$, the former can be converted into the latter by local operations and classical communication (LOCC) if, and only if, $E\left(\left|\psi_{A B}\right\rangle\right) \geq$ $E\left(\left|\phi_{A B}\right\rangle\right)$, where $E$ is the entropy of entanglement [8], given by the von Neumann entropy of either of the two reduced density matrices of the state.

For mixed bipartite states or pure states of more than two parties, however, such a pleasingly simple situation does not hold true anymore. There are examples of mixed bipartite states, known as bound entangled [9], that require a non-zero rate of pure state entanglement for their creation by LOCC in the limit of many copies, but from which no pure state entanglement can be extracted at all [9-11]. As a consequence, in the general case for the manipulation of entanglement by LOCC there is no unique entanglement measure and it is not possible to establish a direct connection to the axiomatic formulation of the second law of thermodynamics.

In this paper we introduce a class of quantum operations that can be considered as the natural counterpart of adiabatic processes in entanglement theory, in the sense that it allows us to formulate a theory of entanglement manipulation with the same structural form as the second law of thermodynamics. The main technical tool for establishing this result is the generalization of quantum Stein's Lemma [13, 14] developed in Ref. [12], which allows us to determine the best strategy and the optimal distinguishability rate for the discrimination of several copies of a given entangled state from an arbitrary sequence of separable states.

Structure: The paper is organized as follows. In section III we motivate the class of quantum operations that we are going to consider for the manipulation of entanglement, while in subsection IA we comment on previous related work. In section III, in turn, we present a few definitions and the main results of the paper. Section IV is devoted to the proof of Theorem $\Pi$ and Corollary III.4. We revisit the choice of the operations employed in section V] Finally, in section VI we discuss the connection of our framework to works on the foundations of the second law of thermodynamics, more specifically to the axiomatic approach of Lieb and Yngvason.

The results in this paper were announced and discussed in Ref. [15] and Ref. [16].

Notation: We let $\mathcal{H}$ be a finite dimensional Hilbert space and $\mathcal{D}(\mathcal{H})$ the set of density operators acting on $\mathcal{H}$. For two states $\rho, \sigma \in \mathcal{D}(\mathcal{H})$, we define the quantum relative entropy of $\rho$ and $\sigma$ as

$$
S(\rho \| \sigma):=\operatorname{tr}(\rho(\log (\rho)-\log (\sigma))) .
$$

Given a Hermitian operator $A,\|A\|_{1}=\operatorname{tr}\left(\sqrt{A^{\dagger} A}\right)$ stands for the trace norm of $A$ and $\operatorname{tr}(A)_{+}$for the trace of the positive part of $A$, i.e. the sum of the positive eigenvalues of $A$. The partial trace of $\rho \in \mathcal{D}\left(\mathcal{H}^{\otimes n}\right)$ with respect to the the $j$-th Hilbert space is denoted by $\operatorname{tr}_{j}(\rho)$. Given a $\mathcal{M} \subseteq \mathbb{R}^{n}$ we define its associate cone by $\operatorname{cone}(\mathcal{M}):=\left\{x: x=\lambda y, y \in \mathcal{M}, \lambda \in \mathbb{R}_{+}\right\}$and its dual cone 
by $\mathcal{M}^{*}:=\left\{x: y^{T} x \geq 0 \forall y \in \mathcal{M}\right\}$. The Bachmann-Landau notation $g(n)=o(f(n))$ stands for $\forall k>0, \exists n_{0}: \forall n>n_{0}, g(n) \leq k f(n)$. Logarithms are taken in the base 2 . The $K$-dimensional maximally entangled state is denoted by $\Phi(K):=\sum_{i=1}^{K} \sum_{j=1}^{K}|i, i\rangle\langle j, j| / K$ and we set $\phi_{2}:=\Phi(2)$.

Given a $k$-partite finite dimensional Hilbert space $\mathcal{H}:=\mathcal{H}_{1} \otimes \ldots \otimes \mathcal{H}_{k}$, we say that a state $\sigma \in \mathcal{D}(\mathcal{H})$ is separable if it can be written as

$$
\sigma=\sum_{j} p_{j} \sigma_{1, j} \otimes \ldots \otimes \sigma_{k, j}
$$

for local states $\sigma_{i, j} \in \mathcal{D}\left(\mathcal{H}_{i}\right)$ and a probability distribution $\left\{p_{j}\right\}$ [17]. If a state is not separable, we say it is entangled. The set of separable states over $\mathcal{H}$ is denoted by $\mathcal{S}(\mathcal{H})$, or simply $\mathcal{S}$ when the Hilbert space $\mathcal{H}$ is clear from the context.

\section{ASYMPTOTICALLY NON-ENTANGLING OPERATIONS}

Studies on the connections of entanglement theory and thermodynamics date back to the earlier foundational works on the subject [18-22]. There it was noted that the basic postulates of quantum mechanics and the definition of entangled states imply that (a) entanglement cannot be created by local operations and classical communication. It was argued that this should be seen as a basic law of quantum information processing and can be considered as a weak qualitative analogue of the second law of thermodynamics, once we make the identification of entanglement with order and of LOCC maps with adiabatic processes.

Local operations and classical communication are the fundamental class of operations to be considered in the distant lab paradigm, for which the definition of entanglement emerges most naturally. However, in view of principle (a) it is important to note that LOCC is not the largest class that cannot generate entanglement out of separable states. Consider, for instance, the class of separable operations, introduced in Ref. [23]. While it is clear that a separable map cannot generate entanglement, it turns out that there are separable operations which cannot be implemented by LOCC [24].

Are separable maps the largest class of quantum operations that cannot create entanglement? As shown in Ref. [25], this is indeed the case if we allow the use of ancillas. That is, if we require that $\Omega \otimes \mathbb{I}_{d}$, where $\mathbb{I}_{d}$ is a identity map which is applied to a $d$-dimensional ancilla state, does not generate entanglement for an arbitrary $d$, then $\Omega$ must be a separable superoperator. However, for the following it will be important to note that there is yet a larger class of operations for which no entanglement can be generated, if we do not require that our class of quantum maps is closed under tensoring with the identity as above.

Definition II.1 Let $\Omega: \mathcal{D}\left(\mathbb{C}^{d_{1}} \otimes \ldots \otimes \mathbb{C}^{d_{m}}\right) \rightarrow \mathcal{D}\left(\mathbb{C}^{d_{1}^{\prime}} \otimes \ldots \otimes \mathbb{C}^{d_{m}^{\prime}}\right)$ be a quantum operation. We say that $\Omega$ is a separability-preserving or a non-entangling map if for every separable state $\sigma \in \mathcal{D}\left(\mathbb{C}^{d_{1}} \otimes \ldots \otimes \mathbb{C}^{d_{m}}\right)$, $\Omega(\sigma)$ is a separable state. We denote the class of such maps by SEPP.

From its very definition, $S E P P$ is the largest class of operations which cannot create entanglement. An example of a completely positive map which is separability-preserving, yet is not a separable operation is the swap operator. In fact, the class $S E P P$ is even strictly larger than the convex hull of separable operations and the composition of separable operations with the swap operator [26].

We can formulate a quantitative version of (a), stating that (b) entanglement cannot be increased by local operations and classical communication. Although (b) is clearly stronger than the first version 
discussed, it is not as fundamental as (a), because we must assume there is an underlying way to quantify entanglement, something that cannot be done in a completely unambiguous manner. Here we will focus on two specific entanglement measures as the quantitative notion of entanglement needed for (b). The first is the relative entropy of entanglement [19, 27], defined as

$$
E_{R}(\rho):=\min _{\sigma \in \mathcal{S}} S(\rho \| \sigma),
$$

where $\mathcal{S}$ is the set of separable states. The second is the (global) robustness of entanglement [28, 29], given by

$$
R_{G}(\rho)=\min _{\sigma \in \mathcal{D}, s \in \mathbb{R}_{+}}\left[s: \frac{\rho+s \sigma}{1+s} \in \mathcal{S}\right] .
$$

We choose these two measures because, using them to quantify entanglement, LOCC is again not the largest class of operations for which (b) is true. Indeed, non-entangling maps are once more the largest such class.

As we will focus on entanglement manipulation in the limit of arbitrarily many copies of the state, we can consider an even larger class of maps, which are non-entangling only in the asymptotic limit. We define this class precisely in section III] but here we would like to anticipate that this class is formed by sequences of maps $\left\{\Lambda_{n}\right\}_{n \in \mathbb{N}}$ - each acting on $n$ copies of the individual multipartite Hilbert space - where each $\Lambda_{n}$ generates at most an $\epsilon_{n}$ amount of entanglement, and such that $\epsilon_{n}$ goes to zero when $n$ grows.

The motivation for identifying the largest class of operations which cannot create entanglement is that we would like to have a class of operations which is as powerful as possible, in order to allow for a simple theory of entanglement transformations, but also one which does not trivialize the theory, in the sense that every state could converted into another and there would be no point to talk about entanglement anymore. In this context, the class of non-entangling maps, or asymptotically non-entangling maps when we look at the manipulation of many copies of the state, emerges as a very suitable choice.

\section{A. Previous Work and Related Approaches}

In Ref. [30], the applicability of Giles axiomatic approach [5] to entanglement theory was studied. It was shown that for pure state bipartite entanglement the same axioms used in the derivation of the second law of thermodynamics hold true. Therefore, one can derive the uniqueness of the entropy of entanglement following the steps taken by Giles in the derivation of the entropy in the context of the second law [5]. One of Giles postulates is that if two states $A$ and $B$ are both adiabatic accessible from another state $C$, either $A$ is adiabatic accessible to $B$ or vice-versa (if not both) [5]. In Ref. [31], it was pointed out that this property does not hold in asymptotic mixed state entanglement transformations under LOCC, showing the inapplicability of Giles approach in the mixed state scenario.

Various approaches have been considered to enlarge the class of operations in a way that could lead to reversibility of entanglement manipulation under such a set of operations. Two closely related but different routes have been taken here.

A first approach was considered in [32-34]. There, entanglement manipulation was studied under the class of operations that maps every state with a positive partial transpose (PPT) into another PPT state (including the use of ancillas). It was realized in [33] that every state with a nonpositive partial transpose becomes distillable under PPT preserving operations. This eliminates 
the phenomenon of bound entanglement in a qualitative level thereby suggesting the possibility of reversibility in this setting. This was taken as a motivation for further studies, e.g. [34], where it was shown that under PPT maps the antisymmetric states of arbitrary dimension can be reversibly interconverted into pure state entanglement, clearly showing a nontrivial example of mixed state reversibility. Unfortunately, no other example have been found so far and, hence, reversibility under the class of PPT operations remains as an open question. In the multi-partite pure state setting PPT preserving operations are not sufficient to ensure reversibility either [35].

In a second approach one considers every PPT state as a free resource in an LOCC protocol. Then again, every state with a non-positive partial transpose becomes distillable [33]. However, in Ref. [36] it was shown, under some unproven but reasonable assumptions, that in this scenario one still has irreversibility.

The possibility of having reversible transformations of entangled states under enlarged classes of operations was also analysed in Ref. [36]. In this work the authors considered the analogy entanglement-energy, first raised in Refs. [16, 20, 21], complementary to the entanglement-entropy analogy [18, 22] considered here (see [16] for a discussion of the results of this paper in this context), to argue that a fully thermodynamical theory of entanglement could in principle be established even considering the existence of bound entanglement. However, under some assumptions on the properties of an entanglement measure there defined, it was shown that one is unlikely to encounter exactly the setting envisioned. Interestingly, it was proven that if one has reversibility under a class of operations that includes mixing, then the unique measure of entanglement governing state transformations is the regularized relative entropy from the set of states which are closed under the class of operations allowed.

\section{Nice Resources}

There is another line of research which our framework is connected to: the quest for identifying the nice resources of quantum information theory, which allow for a simpler theory over the unassisted case. The idea here is not to consider what resources are useful from the point of view of information processing, but actually the ones that are nice in the sense of leading to a marked simplification in the resource theory under consideration.

The first example of such a nice resource is unlimited entanglement between sender and receiver for communication over a noisy quantum channel. It has been proven in Refs. [37, 38] that this leads to a remarkably simple formula for the quantum and classical capacities (which are actually related by a factor of two), which in this case is in single-letter form, meaning that no regularization is needed, and a direct generalization of Shannon's capacity formula for classical noisy channels.

A more recent example is the use of symmetric side channels for sending quantum information. By the no cloning theorem [39, 40] we know that it is not possible to reliably send quantum information through a channel which distributes the information symmetrically between the receiver and the environment. It has been shown in Refs. [41, 42] that nonetheless such channels are nice resources, as it is possible to derive a single-letter and convex expressions for the symmetricside-channel-assisted quantum and private channel capacities. Such an approach has recently lead to a breakthrough in quantum information theory, as it was used by Smith and Yard to show that the quantum channel capacity is not additive [43].

A third example is of course the use of PPT operations and PPT states in entanglement theory, as discussed in the previous section. 


\section{DEFINITIONS AND MAIN RESULTS}

We start with the following definition of maps that generate a small amount of entanglement.

Definition III.1 Let $\Omega: \mathcal{D}\left(\mathbb{C}^{d_{1}} \otimes \ldots \otimes \mathbb{C}^{d_{m}}\right) \rightarrow \mathcal{D}\left(\mathbb{C}^{d_{1}^{\prime}} \otimes \ldots \otimes \mathbb{C}^{d_{m}^{\prime}}\right)$ be a quantum operation. We say that $\Omega$ is an $\epsilon$-non-entangling (or $\epsilon$-separability-preserving) map if for every separable state $\sigma \in \mathcal{D}\left(\mathbb{C}^{d_{1}} \otimes\right.$ $\left.\ldots \otimes \mathbb{C}^{d_{m}}\right)$,

$$
R_{G}(\Omega(\sigma)) \leq \epsilon .
$$

We denote the set of $\epsilon$-non-entangling maps by $\operatorname{SEPP}(\epsilon)$.

We then define an asymptotically non-entangling operation as given by a sequence of trace-preserving $C P$ maps $\left\{\Lambda_{n}\right\}_{n \in \mathbb{N}}, \Lambda_{n}: \mathcal{D}\left(\left(\mathbb{C}^{d_{1}} \otimes \ldots \otimes \mathbb{C}^{d_{m}}\right)^{\otimes n}\right) \rightarrow \mathcal{D}\left(\left(\mathbb{C}^{d_{1}^{\prime}} \otimes \ldots \otimes \mathbb{C}^{d_{m}^{\prime}}\right)^{\otimes n}\right)$, such that each $\Lambda_{n}$ is $\epsilon_{n}$-non-entangling and $\lim _{n \rightarrow \infty} \epsilon_{n}=0$.

It is worth noting that the use of the global robustness to measure the amount of entanglement generated is not arbitrary. The reason for this choice will be explained in section $\mathrm{V}$.

Having defined the class of maps we are going to use to manipulate entanglement, we can define the cost and distillation functions, in terms of the optimal rate of conversion from and to, respectively, the two qubit maximally entangled state

$$
\phi_{2}=\frac{1}{2} \sum_{i=0}^{1} \sum_{j=0}^{1}|i, i\rangle\langle j, j|
$$

Definition III.2 The entanglement cost under asymptotically non-entangling maps of a state $\rho \in$ $\mathcal{D}\left(\mathbb{C}^{d_{1}} \otimes \ldots \otimes \mathbb{C}^{d_{m}}\right)$ is given by

$$
E_{C}^{a n e}(\rho):=\inf _{\left\{k_{n}, \epsilon_{n}\right\}}\left\{\limsup _{n \rightarrow \infty} \frac{k_{n}}{n}: \lim _{n \rightarrow \infty}\left(\min _{\Lambda_{n} \in S E P P\left(\epsilon_{n}\right)}\left\|\rho^{\otimes n}-\Lambda_{n}\left(\phi_{2}^{\otimes k_{n}}\right)\right\|_{1}\right)=0, \lim _{n \rightarrow \infty} \epsilon_{n}=0\right\},
$$

where the infimum is taken over all sequences of integers $\left\{k_{n}\right\}$ and real numbers $\left\{\epsilon_{n}\right\}$. In the formula above $\phi_{2}^{\otimes k_{n}}$ stands for $k_{n}$ copies of a two-dimensional maximally entangled state shared by the first two parties and the maps $\Lambda_{n}: \mathcal{D}\left(\left(\mathbb{C}^{2} \otimes \mathbb{C}^{2}\right)^{\otimes k_{n}}\right) \rightarrow \mathcal{D}\left(\left(\mathbb{C}^{d_{1}} \otimes \ldots \otimes \mathbb{C}^{d_{m}}\right)^{\otimes n}\right)$ are $\epsilon_{n}$-non-entangling operations.

Definition III.3 The distillable entanglement under asymptotically non-entangling maps of a state $\rho \in$ $\mathcal{D}\left(\mathbb{C}^{d_{1}} \otimes \ldots \otimes \mathbb{C}^{d_{m}}\right)$ is given by

$$
E_{D}^{\text {ane }}(\rho):=\sup _{\left\{k_{n}, \epsilon_{n}\right\}}\left\{\liminf _{n \rightarrow \infty} \frac{k_{n}}{n}: \lim _{n \rightarrow \infty}\left(\min _{\Lambda_{n} \in S E P P\left(\epsilon_{n}\right)}\left\|\Lambda_{n}\left(\rho^{\otimes n}\right)-\phi_{2}^{\otimes k_{n}}\right\|_{1}\right)=0, \lim _{n \rightarrow \infty} \epsilon_{n}=0\right\},
$$

where the infimum is taken over all sequences of integers $\left\{k_{n}\right\}$ and real numbers $\left\{\epsilon_{n}\right\}$.

Note that when we do not specify the state of the other parties we mean that their state is trivial. Note furthermore that the fact that initially only two parties share entanglement is not a problem as the class of operations we employ include the swap operation. We are now in the position to state the main result of the paper.

Theorem I For every multipartite state $\rho \in \mathcal{D}\left(\mathbb{C}^{d_{1}} \otimes \ldots \otimes \mathbb{C}^{d_{m}}\right)$,

$$
E_{C}^{a n e}(\rho)=E_{D}^{a n e}(\rho)=E_{R}^{\infty}(\rho):=\lim _{n \rightarrow \infty} \frac{E_{R}\left(\rho^{\otimes n}\right)}{n} .
$$


We note that in Ref. [44] it was shown that in general $E_{R}(\rho \otimes \rho)<2 E_{R}(\rho)$. Therefore the limit in the definition of the regularized quantity $E_{R}^{\infty}$ is necessary.

We find from Theorem \ that under asymptotically non-entangling operations, entanglement can be interconverted reversibly. From this we can readily show that in this setting there is a total order of entangled states.

Corollary III.4 For two multipartite states $\rho \in \mathcal{D}\left(\mathbb{C}^{d_{1}} \otimes \ldots \otimes \mathbb{C}^{d_{m}}\right)$ and $\sigma \in \mathcal{D}\left(\mathbb{C}^{d_{1}^{\prime}} \otimes \ldots \otimes \mathbb{C}^{d^{\prime}}\right)$, there is a sequence of quantum operations $\Lambda_{n}$ such that

$$
\Lambda_{n} \in \operatorname{SEPP}\left(\epsilon_{n}\right), \quad \lim _{n \rightarrow \infty} \epsilon_{n}=0
$$

and

$$
\lim _{n \rightarrow \infty}\left\|\Lambda_{n}\left(\rho^{\otimes n}\right)-\sigma^{\otimes n-o(n)}\right\|_{1}=0
$$

if, and only if,

$$
E_{R}^{\infty}(\rho) \geq E_{R}^{\infty}(\sigma)
$$

We have also identified the regularized relative entropy of entanglement $E_{R}^{\infty}$ as the unique entanglement measure in this framework. As shown in Ref. [12] and discussed in section[IV] this measure is related to the optimal rate of discrimination from many copies of an entangled state to a separable states. Therefore, under asymptotically non-entangling operations the amount of entanglement of any multipartite state is completely determined by how distinguishable the latter is from a state that only contains classical correlations. Furthermore, we showed in Corollary III.3 of [12] that

$$
L G(\rho):=\inf _{\left\{\epsilon_{n}\right\}}\left\{\limsup _{n \rightarrow \infty} \frac{1}{n} L R_{G}^{\epsilon_{n}}\left(\rho^{\otimes n}\right): \lim _{n \rightarrow \infty} \epsilon_{n}=0\right\}=E_{R}^{\infty}(\rho),
$$

where $L R_{G}(\rho):=\log \left(1+R_{G}(\rho)\right)$ is the $\log$ (global) robustness of entanglement [45, 46] and

$$
L R_{G}^{\epsilon}(\rho):=\min _{\tilde{\rho} \in B_{\epsilon}(\rho)} L R_{G}(\tilde{\rho}),
$$

with $B_{\epsilon}(\rho):=\left\{\tilde{\rho} \in \mathcal{D}(\mathcal{H}):\|\rho-\tilde{\rho}\|_{1} \leq \epsilon\right\}$. Hence, we find that the amount of entanglement may equivalently and uniquely be defined in terms of the robustness of quantum correlations to noise in the form of mixing. This observation, in particular Eq. (6), will be important in the proof of Theorem I.

\section{PROOF OF THEOREM [}

As mentioned before, the main technical tool for proving Theorem \is an extension of quantum Stein's Lemma [13, 14], which appeared in Ref. [12] as Theorem I. Here we state the theorem in the particular case of distinguishing a given entangled state from separable states, which is sufficient for our purposes.

Theorem II [12] Let $\rho \in \mathcal{D}(\mathcal{H})$ be an entangled state.

Direct part: For every $\epsilon>0$ there exists a sequence of POVMs $\left\{A_{n}, \mathbb{I}-A_{n}\right\}_{n \in \mathbb{N}}$ such that

$$
\lim _{n \rightarrow \infty} \operatorname{tr}\left(\left(\mathbb{I}-A_{n}\right) \rho^{\otimes n}\right)=0
$$


and for every $n \in \mathbb{N}$ and every separable state $\omega_{n} \in \mathcal{D}\left(\mathcal{H}^{\otimes n}\right)$,

$$
-\frac{\log \operatorname{tr}\left(A_{n} \omega_{n}\right)}{n}+\epsilon \geq E_{R}^{\infty}(\rho) .
$$

Strong converse: For $\epsilon>0$ and sequence of POVMs $\left\{A_{n}, \mathbb{I}-A_{n}\right\}_{n \in \mathbb{N}}$ satisfying

$$
-\frac{\log \left(\operatorname{tr}\left(A_{n} \omega_{n}\right)\right)}{n}-\epsilon \geq E_{R}^{\infty}(\rho)
$$

for every $n \in \mathbb{N}$ and every separable state $\omega_{n} \in \mathcal{D}\left(\mathcal{H}^{\otimes n}\right)$,

$$
\lim _{n \rightarrow \infty} \operatorname{tr}\left(\left(\mathbb{I}-A_{n}\right) \rho^{\otimes n}\right)=1 .
$$

According to Proposition III.1 of [] we can express the statement above as follows.

$$
\lim _{n \rightarrow \infty} \min _{\omega_{n} \in \mathcal{S}\left(\mathcal{H}^{\otimes n}\right)} \operatorname{tr}\left(\rho^{\otimes n}-2^{y n} \omega_{n}\right)_{+}= \begin{cases}0, & y>E_{R}^{\infty}(\rho), \\ 1, & y<E_{R}^{\infty}(\rho) .\end{cases}
$$

From this theorem one can already gain an idea of how we are going to construct asymptotically non-entangling maps for the creation and distillation processes with a rate matching $E_{R}^{\infty}(\rho)$. For entanglement distillation, we consider a sequence of measure-and-prepare quantum operations, which first measure the optimal two-outcome POVM from the direct part of Theorem II, subsequently either preparing approximately $n E_{R}^{\infty}(\rho)$ copies of $\phi_{2}$, following the outcome associated to $\mathbb{I}-A_{n}$ corresponding to $\rho^{\otimes n}$, or the separable state orthogonal to the maximally entangled state for the outcome $A_{n}$ corresponding to a separable state. A simple analysis, performed explicitly in section IVB, shows that this family of maps is indeed asymptotically non-entangling and distills $\phi_{2}$ from $\rho$ with any rate smaller than $E_{R}^{\infty}(\rho)$.

For the entanglement cost of $\rho$ in terms of $\phi_{2}$, we use a similar construction. We again perform a two outcome POVM, but now to check whether we have $n$ copies of $\phi_{2}$ or a state orthogonal to it. For the case corresponding to a maximally entangled state, we then prepare a good approximation $\rho_{n}$ of approximately $n E_{R}^{\infty}(\rho)$ copies of $\rho$, while in the other case we prepare a state which, when mixed with $\rho_{n}$, has the smallest amount of entanglement possible. From the converse part of Theorem II (which implies in particular Eq. 6 [12]), we show in section IVA that the maps are asymptotically non-entangling and create $\rho$ from $\phi_{2}$ with any rate bigger than the regularized relative entropy of entanglement of $\rho$.

It is intriguing that the strong converse part of Theorem $\amalg$ not only implies that distillation with a rate higher than $E_{R}^{\infty}(\rho)$ is impossible, but also that the reverse process, the formation of $\rho$ from $\phi_{2}$, is achievable with any such a rate.

\section{A. The Entanglement Cost under Asymptotically non-Entangling Maps}

We start by showing that the entanglement quantified by the log global robustness cannot increase by more than a factor proportional to $\log (1+\epsilon)$ under $\epsilon$-non-entangling maps.

Lemma IV.1 If $\Lambda \in S E P P(\epsilon)$, then

$$
L R_{G}(\Lambda(\rho)) \leq \log (1+\epsilon)+L R_{G}(\rho) .
$$


Proof Let $\pi$ be an optimal state for $\rho$ achieving $R_{G}(\rho)$

$$
\rho+R_{G}(\rho) \pi=\left(1+R_{G}(\rho)\right) \sigma
$$

where $\sigma$ is a separable state. We have that

$$
\Lambda(\rho)+R_{G}(\rho) \Lambda(\pi)=\left(1+R_{G}(\rho)\right) \Lambda(\sigma)
$$

with $R_{G}(\Lambda(\sigma)) \leq \epsilon$. Setting $Z$ to be a state for which $\Lambda(\sigma)+\epsilon Z$ is separable, we find

$$
\Lambda(\rho)+R_{G}(\rho) \Lambda(\pi)+\epsilon\left(1+R_{G}(\rho)\right) Z=\left(1+R_{G}(\rho)\right) \Lambda(\sigma)+\epsilon\left(1+R_{G}(\rho)\right) Z \in \operatorname{cone}(\mathcal{S}),
$$

from which Eq. (8) follows.

Proposition IV.2 For every multipartite state $\rho \in \mathcal{D}\left(\mathbb{C}^{d_{1}} \otimes \ldots \otimes \mathbb{C}^{d_{2}}\right)$,

$$
E_{C}^{a n e}(\rho)=E_{R}^{\infty}(\rho)
$$

Proof Let $\Lambda_{n} \in \operatorname{SEPP}\left(\epsilon_{n}\right)$ be an optimal sequence of maps for the entanglement cost under asymptotically non-entangling maps, i.e.

$$
\lim _{n \rightarrow \infty}\left\|\Lambda_{n}\left(\phi_{2}^{\otimes k_{n}}\right)-\rho^{\otimes n}\right\|_{1}=0, \quad \lim _{n \rightarrow \infty} \epsilon_{n}=0,
$$

and

$$
\limsup _{n \rightarrow \infty} \frac{k_{n}}{n}=E_{C}^{a n e}(\rho)
$$

Then, from LemmaIV.1,

$$
\begin{aligned}
\frac{1}{n} L R_{G}\left(\Lambda_{n}\left(\phi_{2}^{\otimes k_{n}}\right)\right) & \leq \frac{1}{n} L R_{G}\left(\phi_{2}^{\otimes k_{n}}\right)+\frac{1}{n} \log \left(1+\epsilon_{n}\right) \\
& =\frac{k_{n}}{n}+\frac{1}{n} \log \left(1+\epsilon_{n}\right),
\end{aligned}
$$

where the last equality follows from $R_{G}\left(\phi_{2}^{\otimes k_{n}}\right)=2^{k_{n}}-1$. Hence, as $\lim _{n \rightarrow \infty} \epsilon_{n}=0$,

$$
\begin{aligned}
E_{R}^{\infty}(\rho)=L G(\rho) & \leq \limsup _{n \rightarrow \infty} \frac{1}{n} L R_{G}\left(\Lambda_{n}\left(\phi_{2}^{\otimes k_{n}}\right)\right) \\
& \leq \limsup _{n \rightarrow \infty}\left(\frac{k_{n}}{n}+\frac{1}{n} \log \left(1+\epsilon_{n}\right)\right) \\
& =E_{C}^{\text {ane }}(\rho) .
\end{aligned}
$$

To show the converse inequality, assume w.l.o.g. that $\rho$ is entangled. We consider maps of the form

$$
\Lambda_{n}(A)=\operatorname{tr}\left(A \Phi\left(K_{n}\right)\right) \rho_{n}+\operatorname{tr}\left(A\left(\mathbb{I}-\Phi\left(K_{n}\right)\right)\right) \pi_{n},
$$

where (i) $\left\{\rho_{n}\right\}$ is an optimal sequence of approximations for $\rho^{\otimes n}$ achieving the infimum in $L G(\rho)$ (note the infimum might not be achievable by any sequence $\left\{\rho_{n}\right\}$. In this case, for every $\mu>0$ we can find a sequence $\left\{\rho_{n}^{\mu}\right\}$ such that $\lim _{n \rightarrow \infty} \frac{L R_{G}\left(\rho_{n}^{\mu}\right)}{n}=L G(\rho)+\mu$, proceed as in the case 
where the infimum can be achieved and let $\mu \rightarrow 0$ in the end, obtaining the same results), (ii) $\log \left(K_{n}\right)=\left\lceil\log \left(1+R_{G}\left(\rho_{n}\right)\right)\right\rceil$, and (iii) $\pi_{n}$ is a state such that

$$
\frac{\rho_{n}+\left(K_{n}-1\right) \pi_{n}}{K_{n}} \in \mathcal{S},
$$

which always exists as $K_{n} \geq 2^{\log \left(1+R_{G}\left(\rho_{n}\right)\right)}=1+R_{G}\left(\rho_{n}\right)$. As $\pi_{n}$ and $\rho_{n}$ are states, each $\Lambda_{n}$ is completely positive and trace-preserving.

The next step is to show that each $\Lambda_{n}$ is a $1 /\left(K_{n}-1\right)$-separability-preserving map. From Eq. (10) we find

$$
\frac{\pi_{n}+\left(K_{n}-1\right)^{-1} \rho_{n}}{1+\left(K_{n}-1\right)^{-1}} \in \mathcal{S}
$$

and, thus,

$$
R_{G}\left(\pi_{n}\right) \leq \frac{1}{K_{n}-1}
$$

From Eq. (10) we have that

$$
\Lambda_{n}\left(I_{b}\right)=\frac{\rho_{n}+\left(K_{n}-1\right) \pi_{n}}{K_{n}} \in \mathcal{S}
$$

where $I_{b}$ is the separable isotropic state $I_{b}=\frac{1}{K} \Phi(K)+\frac{\mathbb{I}-\Phi(K)}{K(K+1)}$ at the boundary of the separable states set, and

$$
R_{G}\left(\Lambda_{n}\left(\frac{\mathbb{I}-\Phi\left(K_{n}\right)}{K_{n}^{2}-1}\right)\right)=R_{G}\left(\pi_{n}\right) \leq \frac{1}{K_{n}-1}
$$

From the form of $\Lambda_{n}$ we can w.l.o.g. restrict our attention to isotropic separable input states. Any such state $I(q)$ can be written as

$$
I(q)=q I_{b}+(1-q) \frac{\mathbb{I}-\Phi(K)}{K^{2}-1},
$$

with $0 \leq q \leq 1$. From the convexity of $R_{G}$,

$$
R_{G}\left(\Lambda_{n}(I(q))\right) \leq q R_{G}\left(\Lambda_{n}\left(I_{b}\right)\right)+(1-q) R_{G}\left(\Lambda_{n}\left(\frac{\mathbb{I}-\Phi(K)}{K^{2}-1}\right)\right) \leq \frac{1}{K_{n}-1},
$$

where we used Eq. (11) and

$$
R_{G}\left(\Lambda_{n}\left(I_{b}\right)\right)=0
$$

We hence see that indeed $\Lambda_{n}$ is a $1 /\left(K_{n}-1\right)$-separability-preserving map.

In Corollary II.1 of Ref. [12], it was proven that $E_{R}^{\infty}(\rho)>0$ for every entangled state $\rho$. From Eq. (6) we then find that $L G(\rho)=E_{R}^{\infty}(\rho)>0$ for every entangled state. Therefore

$$
\lim _{n \rightarrow \infty} \frac{1}{K_{n}-1} \leq \lim _{n \rightarrow \infty} \frac{1}{R_{G}\left(\rho_{n}\right)}=0
$$

where the last equality follows from Eq. (6). Moreover, as

$$
\lim _{n \rightarrow \infty}\left\|\rho^{\otimes n}-\Lambda_{n}\left(\Phi\left(K_{n}\right)\right)\right\|_{1}=\lim _{n \rightarrow \infty}\left\|\rho^{\otimes n}-\rho_{n}\right\|_{1}=0
$$


it follows that $\left\{\Lambda_{n}\right\}$ is an allowed sequence of maps for $E_{C}^{a n e}(\rho)$ and, thus,

$$
\begin{aligned}
E_{C}^{a n e}(\rho) & \leq \limsup _{n \rightarrow \infty} \frac{1}{n} \log \left(K_{n}\right) \\
& =\limsup _{n \rightarrow \infty} \frac{1}{n}\left\lceil\log \left(1+R_{G}\left(\rho_{n}\right)\right)\right\rceil \\
& =L G(\rho) \\
& =E_{R}^{\infty}(\rho) .
\end{aligned}
$$

\section{B. The Distillable Entanglement under non-Entangling Operations}

Before we turn to the proof of the main proposition of this section, we state and prove an auxiliary lemma which will be used later on. It can be considered the analogue for non-entangling maps of Theorem 3.3 of Ref. [32], which deals with PPT maps.

Lemma IV.3 For every multipartite state $\rho \in \mathcal{D}\left(\mathbb{C}^{d_{1}} \otimes \ldots \otimes \mathbb{C}^{d_{n}}\right)$ the singlet-fraction under nonentangling maps,

$$
F_{\text {sep }}(\rho ; K):=\max _{\Lambda \in S E P P} \operatorname{tr}(\Phi(K) \Lambda(\rho))
$$

where $\Phi(K)$ is a $K$-dimensional maximally entangled state shared by the first two parties, satisfies

$$
F_{\text {sep }}(\rho ; K)=\min _{\sigma \in \operatorname{cone}(\mathcal{S})}\left[\operatorname{tr}(\rho-\sigma)_{+}+\frac{1}{K} \operatorname{tr}(\sigma)\right]
$$

Proof Due to the $U U^{*}$-symmetry of the maximally entangled state and the fact that the composition of a $S E P P$ operation with the twirling map is again a non-entangling operation, we can w.l.o.g. perform the maximization over $S E P P$ maps of the form

$$
\Lambda(\rho)=\operatorname{tr}(A \rho) \Phi(K)+\operatorname{tr}((\mathbb{I}-A) \rho) \frac{\mathbb{I}-\Phi(K)}{K^{2}-1} .
$$

Since $\Lambda$ must be completely positive we have $0 \leq A \leq \mathbb{I}$. As $\Lambda(\rho)$ is an isotropic state for every input state $\rho$, it is separable iff $\operatorname{tr}(\Lambda(\rho) \Phi(K)) \leq 1 / K$ [47]. Hence, we find that $\Lambda$ is non-entangling iff for every separable state $\sigma$,

$$
\operatorname{tr}(A \sigma) \leq \frac{1}{K}
$$

The singlet fraction is thus given by

$$
F_{\text {sep }}(\rho ; K)=\max _{A}[\operatorname{tr}(A \rho): 0 \leq A \leq \mathbb{I}, \quad \operatorname{tr}(A \sigma) \leq 1 / K, \quad \forall \sigma \in \mathcal{S}] .
$$

The R.H.S. of this equation is a convex optimization problem and we can find its dual formulation. Let us form the Lagrangian of the problem,

$$
L(\rho, A, X, Y)=-\operatorname{tr}(A \rho)-\operatorname{tr}(X A)-\operatorname{tr}(Y(\mathbb{I}-A))-\operatorname{tr}((\mathbb{I} / K-A) Z),
$$


where $X, Y \geq 0$ are Lagrange multipliers associated to the constraints $0 \leq A \leq \mathbb{I}$, and $Z \in \operatorname{cone}(\mathcal{S})$ is a Lagrange multiplier (an unnormalized separable state) associated to the constraint $\operatorname{tr}(A \sigma) \leq$ $1 / K \forall \sigma \in \mathcal{S}$. The dual problem is then given by

$$
F_{\text {sep }}(\rho ; K)=\min _{Y, Z}\left[\operatorname{tr}(Y)+\frac{1}{K} \operatorname{tr}(Z): Z \in \operatorname{cone}(\mathcal{S}), Y \geq 0, Y \geq \rho-Z\right] .
$$

Using that $\operatorname{tr}(A)_{+}=\min _{Y \geq A, Y \geq 0} \operatorname{tr}(Y)$, we then find Eq. (13).

It turns out that to demonstrate that distillable entanglement equals the regularized relative entropy of entanglement we do not need to allow any generation of entanglement from the maps. In analogy to Definition $\amalg$ II.3, we can define the distillable entanglement under non-entangling maps as

$$
E_{D}^{n e}(\rho):=\sup _{\left\{k_{n}\right\}}\left\{\liminf _{n \rightarrow \infty} \frac{k_{n}}{n}: \lim _{n \rightarrow \infty}\left(\min _{\Lambda_{n} \in S E P P}\left\|\Lambda_{n}\left(\rho^{\otimes n}\right)-\phi_{2}^{\otimes k_{n}}\right\|_{1}\right)=0\right\}
$$

Using Lemma IV.3 and Theorem $\Pi$ we can easily establish the following proposition.

Proposition IV.4 For every multipartite entangled state $\rho \in \mathcal{D}\left(\mathbb{C}^{d_{1}} \otimes \ldots \otimes \mathbb{C}^{d_{n}}\right)$,

$$
E_{D}^{n e}(\rho)=E_{R}^{\infty}(\rho) .
$$

Proof From Lemma IV.3 we find

$$
F_{\text {sep }}\left(\rho^{\otimes n} ; 2^{n y}\right):=\min _{\sigma \in \mathcal{S}, b \in \mathbb{R}}\left[\operatorname{tr}\left(\rho^{\otimes n}-2^{n b} \sigma\right)_{+}+2^{-(y-b) n}\right] .
$$

Let us consider the asymptotic behavior of $F_{\text {sep }}\left(\rho^{\otimes n}, 2^{n y}\right)$. Take $y=E_{R}^{\infty}(\rho)+\epsilon$, for any $\epsilon>0$. Then we can choose, for each $n, b=n\left(E_{R}^{\infty}(\rho)+\frac{\epsilon}{2}\right)$, giving

$$
F_{\text {sep }}\left(\rho^{\otimes n}, 2^{n y}\right) \leq \min _{\sigma \in \mathcal{S}}\left[\operatorname{tr}\left(\rho^{\otimes n}-2^{n\left(E_{\mathcal{M}}^{\infty}(\rho)+\frac{\epsilon}{2}\right)} \sigma\right)_{+}\right]+2^{-n \frac{\epsilon}{2}} .
$$

We then see from Eq. (7) that $\lim _{n \rightarrow \infty} F_{\text {sep }}\left(\rho^{\otimes n}, 2^{n y}\right)=0$, from which follows that $E_{D}^{n e}(\rho) \leq$ $E_{R}^{\infty}(\rho)+\epsilon$. As $\epsilon$ is arbitrary, we find $E_{D}^{n e}(\rho) \leq E_{R}^{\infty}(\rho)$.

Conversely, let us take $y=E_{R}^{\infty}(\rho)-\epsilon$, for any $\epsilon>0$. The optimal $b$ for each $n$ has to satisfy $b_{n} \leq y$, otherwise $F_{\text {sep }}\left(\rho^{\otimes n}, 2^{n y}\right)$ would be larger than one, which is not true. Therefore,

$$
F_{\text {sep }}\left(\rho^{\otimes n}, 2^{n y}\right) \geq \min _{\sigma \in \mathcal{S}} \operatorname{tr}\left(\rho^{\otimes n}-2^{n\left(E_{R}^{\infty}(\rho)-\epsilon\right)} \sigma\right)_{+},
$$

which, by Eq. (7), tends to unity again. This then shows that $E_{D}^{n e}(\rho) \geq E_{R}^{\infty}(\rho)-\epsilon$. Again, as $\epsilon>0$ is arbitrary, we find $E_{D}^{n e}(\rho) \geq E_{R}^{\infty}(\rho)$.

The proof of the other half of Theorem【 follows easily from PropositionIV.4 and the following Lemma.

Lemma IV.5 If $\Lambda \in S E P P(\epsilon, \mathcal{H})$, then

$$
E_{R}(\Lambda(\rho)) \leq \log (1+\epsilon)+E_{R}(\rho) .
$$


Proof Let $\sigma$ be an optimal separable state for $\rho$ in the relative entropy of entanglement. Then, if $\Lambda$ is a $\epsilon$-separability preserving map and $Z$ a state such that $\Lambda(\sigma)+\epsilon Z$ is separable,

$$
\begin{aligned}
E_{R}(\rho) & =S(\rho \| \sigma) \\
& \geq S(\Lambda(\rho) \| \Lambda(\sigma)) \\
& \geq S(\Lambda(\rho) \| \Lambda(\sigma)+\epsilon Z) \\
& =S(\Lambda(\rho) \|(\Lambda(\sigma)+\epsilon Z) /(1+\epsilon))-\log (1+\epsilon) \\
& \geq E_{R}(\Lambda(\rho))-\log (1+\epsilon),
\end{aligned}
$$

The first inequality follows from the monotonicity of the relative entropy under trace preserving $\mathrm{CP}$ maps and the second inequality from the operator monotonicity of the log.

Indeed, as any sequence of non-entangling maps is obviously asymptotically non-entangling, we have $E_{D}^{a n e}(\rho) \geq E_{D}^{n e}(\rho)=E_{R}^{\infty}(\rho)$, where the last equality follows from Proposition IV.4. To prove the converse inequality $E_{D}^{a n e}(\rho) \leq E_{R}^{\infty}(\rho)$, we use LemmaIV.5, Let $\Lambda_{n} \in S E P P\left(\epsilon_{n}\right)$ be an optimal sequence of maps for the distillable entanglement under asymptotically non-entangling maps in the sense that

$$
\lim _{n \rightarrow \infty}\left\|\Lambda_{n}\left(\rho^{\otimes n}\right)-\phi_{2}^{\otimes k_{n}}\right\|_{1}=0 \quad \lim _{n \rightarrow \infty} \epsilon_{n}=0
$$

and

$$
\liminf _{n \rightarrow \infty} \frac{k_{n}}{n}=E_{D}^{a n e}(\rho)
$$

From LemmaIV.5,

$$
\frac{1}{n} E_{R}\left(\Lambda_{n}\left(\rho^{\otimes n}\right)\right) \leq \frac{1}{n} E_{R}\left(\rho^{\otimes n}\right)+\frac{1}{n} \log \left(1+\epsilon_{n}\right) .
$$

Hence, as $\lim _{n \rightarrow \infty} \epsilon_{n}=0$ and from the asymptotic continuity of relative entropy of entanglement,

$$
\begin{aligned}
E_{D}^{\text {ane }}(\rho) & =\liminf _{n \rightarrow \infty} \frac{1}{n} E_{R}\left(\Lambda_{n}\left(\rho^{\otimes n}\right)\right) \\
& \leq \liminf _{n \rightarrow \infty} \frac{1}{n} E_{R}\left(\rho^{\otimes n}\right)+\liminf _{n \rightarrow \infty} \frac{1}{n} \log \left(1+\epsilon_{n}\right) \\
& =E_{R}^{\infty}(\rho) .
\end{aligned}
$$

\section{Proof Corollary III.4}

Finally, we can now easily establish Corollary ஹI.4.

Proof (Corollary III.4) We assume w.l.o.g. that $\sigma$ is entangled. Then, by Corollary ... of [], $E_{R}^{\infty}(\sigma)>$ 0 .

First, let us assume there is a sequence of quantum maps $\left\{\Lambda_{n}\right\}_{n \in \mathbb{N}}$ satisfying the three conditions of the corollary. Then,

$$
\begin{aligned}
E_{R}^{\infty}(\sigma) & =\lim _{n \rightarrow \infty} \frac{1}{n} E_{R}\left(\Lambda_{n}\left(\rho^{\otimes n}\right)\right) \\
& \leq \frac{1}{n} E_{R}\left(\rho^{\otimes n}\right)+\frac{\log \left(1+\epsilon_{n}\right)}{n} \\
& =E_{R}^{\infty}(\rho) .
\end{aligned}
$$


The first equality follow from the asymptotic continuity of $E_{R}$ [48] and the following inequality from LemmaIV.5.

To show the other direction, let us assume that $E_{R}^{\infty}(\rho) \geq E_{R}^{\infty}(\sigma)$. As $E_{R}^{\infty}(\rho)=E_{D}^{a n e}(\rho)$, there is a sequence of maps $\left\{\Lambda_{n}\right\}_{n \in \mathbb{N}}, \Lambda_{n}: \mathcal{D}\left(\left(\mathbb{C}^{d_{1}} \otimes \ldots \otimes \mathbb{C}^{d_{m}}\right)^{\otimes n}\right) \rightarrow \mathcal{D}\left(\left(\mathbb{C}^{2} \otimes \mathbb{C}^{2}\right)^{\otimes k_{n}}\right)$, such that

$$
\begin{gathered}
\Lambda_{n} \in \operatorname{SEPP}\left(\epsilon_{n}\right), \quad \lim _{n \rightarrow \infty} \epsilon_{n}=0, \\
\lim _{n \rightarrow \infty}\left\|\Lambda_{n}\left(\rho^{\otimes n}\right)-\phi_{2}^{\otimes k_{n}}\right\|_{1}=0
\end{gathered}
$$

and

$$
\lim _{n \rightarrow \infty} \frac{k_{n}}{n}=E_{R}^{\infty}(\rho)
$$

Note we can always find a sequence for which the limit in Eq. (18) exists by using the optimal sequence such that $\limsup _{n \rightarrow \infty} \frac{k_{n}}{n}=E_{R}^{\infty}(\rho)$ and increasing the value of the $k_{n}$ 's which are not close to the limit value.

Moreover, as $E_{R}^{\infty}(\sigma)=E_{C}^{a n e}(\sigma)$, there is another sequence of maps $\left\{\Omega_{n}\right\}_{n \in \mathbb{N}}, \Omega_{n}: \mathcal{D}\left(\left(\mathbb{C}^{2} \otimes\right.\right.$ $\left.\left.\mathbb{C}^{2}\right)^{\otimes k_{n}^{\prime}}\right) \rightarrow \mathcal{D}\left(\left(\mathbb{C}^{d_{1}^{\prime}} \otimes \ldots \otimes \mathbb{C}^{d^{\prime}{ }^{\prime}}\right)^{\otimes n}\right)$, satisfying

$$
\begin{gathered}
\Omega_{n} \in \operatorname{SEPP}\left(\epsilon_{n}^{\prime}\right), \quad \lim _{n \rightarrow \infty} \epsilon_{n}^{\prime}=0, \\
\lim _{n \rightarrow \infty}\left\|\Omega_{n}\left(\phi_{2}^{\otimes k_{n}^{\prime}}\right)-\sigma^{\otimes n}\right\|_{1}=0
\end{gathered}
$$

and

$$
\lim _{n \rightarrow \infty} \frac{k_{n}^{\prime}}{n}=E_{R}^{\infty}(\sigma)
$$

From Eqs. (18) and (19) there is a sequence $\delta_{n_{0}}$ converging to zero when $n_{0} \rightarrow \infty$ such that for every $n \geq n_{0}$,

$$
k_{n} \geq\left(E_{R}^{\infty}(\rho)-\delta_{n_{0}} / 2\right) n, \quad k_{n}^{\prime} \leq\left(E_{R}^{\infty}(\sigma)+\delta_{n_{0}} / 2\right) n .
$$

Then, for every $n \geq n_{0}, k_{n} \geq-\delta_{n_{0}} n+k_{n}^{\prime}$. From Eq. (19) we thus find that for sufficiently large $n \geq n_{0}$,

$$
k_{n}=k_{n-o(n)}^{\prime}+r_{n}
$$

with $r_{n}$ a positive integer.

Let us now consider the sequence of maps $\left\{\Omega_{n} \circ \operatorname{tr}_{1, \ldots, r_{n}} \circ \Lambda_{n}\right\}_{n \in \mathbb{N}}$. From Eqs. (18, 19) and the fact that the trace-norm contracts under completely positive trace-preserving maps we find

$$
\begin{aligned}
\lim _{n \rightarrow \infty}\left\|\Omega_{n-o(n)} \circ \operatorname{tr}_{1, \ldots, r_{n}} \circ \Lambda_{n}\left(\rho^{\otimes n}\right)-\sigma^{\otimes n-o(n)}\right\|_{1} & \leq \lim _{n \rightarrow \infty}\left\|\Lambda_{n}\left(\rho^{\otimes n}\right)-\phi_{2}^{\otimes k_{n}}\right\|_{1} \\
& +\left\|\Omega_{n-o(n)}\left(\phi_{2}^{\otimes k_{n-o(n)}^{\prime}}\right)-\sigma^{\otimes n-o(n)}\right\|_{1}=0 .
\end{aligned}
$$

Moreover, from LemmaIV.1 we see that for every separable state $\sigma$,

$$
\begin{aligned}
L R_{G}\left(\Omega_{n-o(n)} \circ \operatorname{tr}_{1, \ldots, r_{n}} \circ \Lambda_{n}(\sigma)\right) & \leq L R_{G}\left(\Lambda_{n}(\sigma)\right)+\log \left(1+\epsilon_{n}^{\prime}\right) \\
& \leq \log \left(1+\epsilon_{n}\right)+\log \left(1+\epsilon_{n}^{\prime}\right),
\end{aligned}
$$




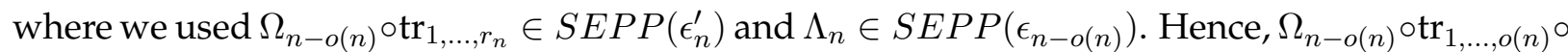
$\Lambda_{n} \in S E P P\left(\epsilon_{n}+\epsilon_{n}^{\prime}+\epsilon_{n} \epsilon_{n}^{\prime}\right)$.

Remark: The structure of the proof can be applied to other situations apart from entanglement conversion. First, as discussed in Ref. [12], Theorem II holds true not only to discrimination of an entangled state from a sequence of separable states, but also to the discrimination of any i.i.d. quantum state from a sequence of states belonging to sets $\mathcal{M}_{n}$, satisfying five certain properties (see Ref. [12] for details). In addition to Theorem [II, the only particular property of entangled states that we used is that (i) $\Phi(K)$ is entangled; (ii) the largest fidelity of $\Phi(K)$ with a separable state is $1 / K$; an (iii) isotropic states (convex combinations of $\Phi(K)$ and its orthogonal state) are separable iff the weight of $\Phi(K)$ is smaller than $1 / K$. Therefore, Theorem $\Pi$ is true in other settings, as long as the properties mentioned before remain true if we change the set of separable states for another one. For example, we can find similar conclusions for a conversion theory of states with a non-positive partial transpose, where PPT states are considered in the place of separable states.

\section{HOW MUCH ENTANGLEMENT MUST AND CAN BE GENERATED?}

We are now in position to understand the choice of the global robustness as the measure to quantify the amount of entanglement generated. The reason that we need to allow some entanglement to be generated is that we relate the entanglement cost to the regularized relative entropy of entanglement by using the connection of the latter to the asymptotic global robustness. The amount of entanglement generated is then due to the fact that the optimal mixing state in the global robustness might be entangled. Before we analyse more carefully if we indeed need to allow for some entanglement to be generated, let us analyse if we can quantify it by some other measure, instead of the global robustness.

Suppose we required alternatively only that

$$
\lim _{n \rightarrow \infty} \max _{\sigma \in \mathcal{S}} \min _{\pi \in \mathcal{S}}\left\|\Lambda_{n}(\sigma)-\pi\right\|_{1}=0,
$$

instead of $\lim _{n \rightarrow \infty} \max _{\sigma \in \mathcal{S}} R_{G}\left(\Lambda_{n}(\sigma)\right)=0$. Then the achievability part in Proposition IV.4 would still hold, as we use operations which do not generate any entanglement, i.e. they map separable states to separable states.

However this is not sufficient. We still have to make sure that the cost is larger than the distillation function, which should be finite. It is easy to see that Eq. (20) ensures that both the distillation and cost functions are zero for separable states. It turns out however that the distillable entanglement is infinite for every entangled state! We hence have a bizarre situation in which even though entanglement cannot be created for free, it can be amplified to the extreme whenever present, no matter in what amount. The key to see this is to consider the analogue of $F_{s e p}$, given by Eq. (12), when we only require that the map satisfies Eq. 20. Following the proof of Lemma IV.3 we can easily see that the singlet-fraction under maps $\Lambda$ satisfying

$$
\max _{\sigma \in \mathcal{S}} \min _{\pi \in \mathcal{S}}\|\Lambda(\sigma)-\pi\|_{1} \leq \epsilon
$$

is given by

$$
F_{\text {sep }}(\rho ; K ; \epsilon)=\min _{\sigma \in \operatorname{cone}(\mathcal{S})}\left[\operatorname{tr}(\rho-\sigma)_{+}+\operatorname{tr}(\sigma)\left(\frac{1}{K}+\epsilon\right)\right],
$$

which for $\rho^{\otimes n}$ can be rewritten as

$$
F_{\text {sep }}\left(\rho^{\otimes n} ; 2^{n y} ; \epsilon_{n}\right)=\min _{\sigma \in \mathcal{S}, b \in \mathbb{R}}\left[\operatorname{tr}\left(\rho^{\otimes n}-2^{b n} \sigma\right)_{+}+2^{-(y-b) n}+2^{-\left(\left(\log \left(1 / \epsilon_{n}\right) / n\right)-b\right) n}\right] .
$$


It is clear that the optimal $b$ must be such that $b<\min \left(y, \log \left(1 / \epsilon_{n}\right) / n\right)$, as otherwise $F_{\text {sep }}\left(\rho^{\otimes n} ; 2^{n y} ; \epsilon\right)$ would be larger than unity. Therefore, if $y>\log \left(1 / \epsilon_{n}\right) / n$,

$$
F_{\text {sep }}\left(\rho^{\otimes n} ; 2^{n y} ; \epsilon_{n}\right) \geq \min _{\sigma \in \mathcal{S}} \operatorname{tr}\left(\rho^{\otimes n}-\epsilon_{n}^{-1} \sigma\right)_{+} \cdot
$$

By Theorem $F_{\text {sep }}\left(\rho^{\otimes n} ; 2^{n y} ; \epsilon_{n}\right)$ approaches unity for every $y$, as long as $\epsilon_{n}$ goes to zero slower than $2^{-n E_{R}^{\infty}(\rho)}$, which implies that the associated distillable entanglement is unbounded. Note that the same happens if we use any asymptotically continuous measure to bound the amount of entanglement generated. Here we denote a measure $E$ is asymptotically continuous if for all states $\rho, \sigma \in \mathcal{D}(\mathcal{H}),|E(\rho)-E(\sigma)| \leq \log (\operatorname{dim}(\mathcal{H})) f\left(\left\|\rho_{n}-\sigma_{n}\right\|_{1}\right)$, for a real valued function $f$ independent of $\operatorname{dim}(\mathcal{H})$ such that $\lim _{x \rightarrow \infty} f(x)=0$.

If instead we require that

$$
\max _{\sigma \in \mathcal{S}} \min _{\pi \in \mathcal{S}}\|\Lambda(\sigma)-\pi\|_{1} \leq \epsilon / \operatorname{dim}(\mathcal{H})
$$

or even that

$$
\max _{\sigma \in \mathcal{S}} \min _{\pi \in \mathcal{S}}\|\Lambda(\sigma)-\pi\|_{\infty} \leq \epsilon / \operatorname{dim}(\mathcal{H}),
$$

then we would find that the associated $\epsilon$-singlet-fraction would satisfy

$$
\tilde{F}_{\text {sep }}(\rho ; K ; \epsilon)=\min _{\sigma \in \operatorname{cone}(\mathcal{S})}\left[\operatorname{tr}(\rho-\sigma)_{+}+\operatorname{tr}(\sigma) \frac{1+\epsilon}{K}\right] .
$$

In this case it is easy to see that the distillable entanglement would be bounded and we would recover a sensible situation. It is interesting and rather mysterious to the authors that although it seems that some entanglement must be generated to have reversibility, only very little can actually be afforded before the theory becomes trivial.

For analysing the necessity of generating some entanglement for reversibility, we consider the following variant of $R_{G}$ [28]:

$$
R(\rho)=\min _{\sigma \in \mathcal{S}, s \in \mathbb{R}}\left[s: \frac{\rho+s \sigma}{1+s} \in \mathcal{S}\right]
$$

and its $\log$ version $L R(\rho):=\log (1+R(\rho))$. Then, in analogy to $L G$, we define

$$
L H(\rho):=\inf _{\left\{\epsilon_{n}\right\}}\left\{\limsup _{n \rightarrow \infty} \frac{1}{n} L R^{\epsilon_{n}}\left(\rho^{\otimes n}\right): \lim _{n \rightarrow \infty} \epsilon_{n}=0\right\}
$$

where

$$
L R^{\epsilon}(\rho):=\min _{\tilde{\rho} \in B_{\epsilon}(\rho)} L R(\tilde{\rho}),
$$

with $B_{\epsilon}(\rho):=\left\{\tilde{\rho} \in \mathcal{D}(\mathcal{H}):\|\rho-\tilde{\rho}\|_{1} \leq \epsilon\right\}$. Following the proof of Proposition IV.2 it is straightforward to show that the entanglement cost under strictly non-entangling maps is given by $L H$. Therefore, the question whether we must allow the generation of some entanglement in order to have a reversible theory reduces to the question whether the two robustness measures $L G$ and $L H$ become the same quantity after smoothing and regularization. 


\section{CONNECTION TO THE AXIOMATIC FORMULATION OF THE SECOND LAW OF THERMODYNAMICS}

In this section we comment on the similarities and differences of entanglement manipulation under asymptotically non-entangling operations and the axiomatic approach of Giles [5] and more particularly of Lieb and Yngvason [6] for the second law of thermodynamics. It must be emphasized that we are comparing the two theories only on an abstract level. Indeed, although the two theories deals with completely different resources (entanglement and order) and have also a distinct range of applicability, we find that the structural form of them both is actually very much related.

Let us start by briefly recalling the axioms used in Ref. [6] in order to derive the second law. Their starting point is the definition of a system as a collection of points called state space and denoted by $\Gamma$. The individual points of a state space are the states of the system. The composition of two state spaces $\Gamma_{1}$ and $\Gamma_{2}$ is given by their Cartesian product. Furthermore, the scaled copies of a given system are defined as follows: if $t>0$ is some fixed number, the state space $\Gamma^{(t)}$ consists of points denoted by $t X$ with $X \in \Gamma$. Finally, a preorder $\prec$ on the state space satisfying the following axioms is assumed:

1. $X \prec X$.

2. $X \prec Y$ and $Y \prec Z$ implies $X \prec Z$.

3. If $X \prec Y$, then $t X \prec t Y$ for all $t>0$.

4. $X \prec(t X,(1-t) X)$ and $(t X,(1-t) X) \prec X$ for all $0 \leq y \leq 1$.

5. If, for some pair of states, $X$ and $Y$,

$$
\left(X, \epsilon Z_{0}\right) \prec\left(Y, \epsilon Z_{1}\right)
$$

holds for a sequence of $\epsilon^{\prime}$ s tending to zero and some states $Z_{0}, Z_{1}$, then $X \prec Y$.

6. $X \prec X^{\prime}$ and $Y \prec Y^{\prime}$ implies $(X, Y) \prec\left(X^{\prime}, Y^{\prime}\right)$.

It was then shown that these axioms, together with the comparison hypothesis, which states that

Comparison Hypothesis: for any two states $X$ and $Y$ in the same state space $\Gamma$, either $X \prec Y$ or

$$
Y \prec X \text {, }
$$

are sufficient to prove the existence of a single valued entropy function completely determining the order induced by the relation $\prec$. More precisely, one also need the vality of the comparison hypothesis for all two-fold scaled products $(1-t) \Gamma \times t \Gamma$ of the state space $\Gamma$.

In the context of entanglement transformations, we interpret the relation $\rho \prec \sigma$ as the possibility of asymptotically transforming $\rho$ into $\sigma$ by asymptotically non-entangling maps. Then, the composite state $(\rho, \sigma)$ is nothing but the tensor product $\rho \otimes \sigma$. Moreover, $t \rho$ takes the form of $\rho^{\otimes t}$. Then $\rho^{\otimes t} \prec \sigma$ expresses the fact that asymptotically $t$ copies of $\rho$ can be transformed into one of $\sigma$. More concretely, we say that

$$
\rho^{\otimes t} \prec \sigma^{\otimes q}
$$


for positive real numbers $t, q$ if there is a sequence of integers $n_{t}, n_{q}$ and of $\operatorname{SEPP}\left(\epsilon_{n}\right) \operatorname{maps} \Lambda_{n}$ such that

$$
\begin{gathered}
\lim _{n \rightarrow \infty}\left\|\Lambda_{n}\left(\rho^{\otimes n_{t}}\right)-\sigma^{\otimes n_{q}-o(n)}\right\|_{1}=0, \\
\lim _{n \rightarrow \infty} \epsilon_{n}=0, \quad \lim _{n \rightarrow \infty} \frac{n_{t}}{n}=t, \quad \text { and } \quad \lim _{n \rightarrow \infty} \frac{n_{q}}{n}=q .
\end{gathered}
$$

With this definition it is straightforward to observe that properties 1, 3, and 4 hold true for entanglement manipulation under asymptotically non-entangling maps. Property 2 can be shown to hold, in turn, by noticing that, from Lemma IV.1 if $\Lambda \in S E P P(\epsilon)$ and $\Omega \in S E P P(\delta)$, then $\Lambda \circ \Omega \in S E P P(\epsilon+\delta+\delta \epsilon)$. Therefore the composition of two asymptotically non-entangling maps is again asymptotically non-entangling. That property 5 is also true is proven in the following lemma.

Lemma VI.1 If for two states $\rho$ and $\sigma$,

$$
\rho \otimes \pi_{1}^{\otimes \epsilon} \prec \sigma \otimes \pi_{2}^{\otimes \epsilon}
$$

holds for a sequence of $\epsilon$ 's tending to zero and two states $\pi_{0}$, $\pi_{1}$, then $\rho \prec \sigma$.

Proof Eq. (23) means that for every $\epsilon>0$ there is a sequence of maps $\Lambda_{n} \in \operatorname{SEPP}\left(\epsilon_{n}\right)$ such that

$$
\begin{gathered}
\lim _{n \rightarrow \infty}\left\|\Lambda_{n}\left(\rho^{\otimes n} \otimes \pi_{1}^{\otimes n_{\epsilon}}\right)-\sigma^{\otimes n-o(n)} \otimes \pi_{2}^{\otimes n_{\epsilon}^{\prime}-o(n)}\right\|_{1}=\lim _{n \rightarrow \infty} \delta_{n}=0, \\
\lim _{n \rightarrow \infty} \epsilon_{n}=0, \quad \lim _{n \rightarrow \infty} \frac{n_{\epsilon}}{n}=\epsilon, \quad \text { and } \quad \lim _{n \rightarrow \infty} \frac{n_{\epsilon}^{\prime}}{n}=\epsilon .
\end{gathered}
$$

We have

$$
\begin{aligned}
\frac{1}{n} E_{R}\left(\rho^{\otimes n}\right)+\frac{1}{n} E_{R}\left(\pi_{1}^{\otimes n_{\epsilon}}\right) & \geq \frac{1}{n} E_{R}\left(\rho^{\otimes n} \otimes \pi_{1}^{\otimes n_{\epsilon}}\right) \\
& \geq \frac{1}{n} E_{R}\left(\Lambda_{n}\left(\rho^{\otimes n} \otimes \pi_{1}^{\otimes n_{\epsilon}}\right)\right)-\frac{\log \left(1+\epsilon_{n}\right)}{n} \\
& \geq \frac{1}{n} E_{R}\left(\sigma^{\otimes n-o(n)} \otimes \pi_{2}^{\otimes n_{\epsilon}^{\prime}-o(n)}\right)-f\left(\delta_{\epsilon}\right)-\frac{\log \left(1+\epsilon_{n}\right)}{n} \\
& \geq \frac{1}{n} E_{R}\left(\sigma^{\otimes n-o(n)}\right)-f\left(\delta_{\epsilon}\right)-\frac{\log \left(1+\epsilon_{n}\right)}{n}
\end{aligned}
$$

where $f: \mathbb{R} \rightarrow \mathbb{R}$ is such that $\lim _{x \rightarrow 0} f(x)=0$. The first inequality follows from the subadditivity of $E_{R}$, the second from Lemma IV.5, the third from the asymptotic continuity of $E_{R}$, and the last from the monotonicity of $E_{R}$ under the partial trace.

As $E_{R}\left(\pi_{2}\right) \leq \log (\operatorname{dim}(\mathcal{H}))$, where $\mathcal{H}$ is the Hilbert space in which $\pi_{2}$ acts on, we find

$$
\frac{1}{n} E_{R}\left(\rho^{\otimes n}\right) \geq \frac{1}{n} E_{R}\left(\sigma^{\otimes n-o(n)}\right)-f\left(\delta_{\epsilon}\right)-\frac{\log \left(1+\epsilon_{n}\right)}{n}-\frac{n_{\epsilon}}{n} \log (\operatorname{dim}(\mathcal{H})) .
$$

Taking the limit $n \rightarrow \infty$,

$$
E_{R}^{\infty}(\rho) \geq E_{R}^{\infty}(\sigma)-\epsilon .
$$

Taking $\epsilon \rightarrow 0$ we find that $E_{R}^{\infty}(\rho) \geq E_{R}^{\infty}(\sigma)$. The Lemma then follows from Corollary III.4. 
The Comparison Hypothesis, in turn, follows from Corollary [II.4 it expresses the total order induced by the regularized relative entropy of entanglement.

We do not know if the theory we are considering for entanglement satisfy axiom 6 . This is fundamentally linked to the possibility of having entanglement catalysis [49] under asymptotically non-entangling transformations. One can prove the following simple lemma.

Lemma VI.2 For entanglement transformations under asymptotically non-entangling maps, axiom 6 is equivalent to

$$
\text { If there is a } \pi \text { such that } \rho \otimes \pi \prec \sigma \otimes \pi \text {, then } \rho \prec \sigma \text {. }
$$

Proof In Theorem 2.1 of Ref. [6] is was shown that axiom 1-6 implies Eq. (24). Since entanglement manipulations under asymptotically non-entangling maps satisfies axioms 1-5, we find one direction of the equivalence.

To prove the converse, assume Eq. (24) holds true. Following [6], we use $X \prec \prec Y$ to denote the situation in which $X \prec Y$, but the reverse transformation is impossible. We claim that Eq. (24) implies

$$
\rho \prec \prec \sigma \Rightarrow \rho \otimes \pi \prec \sigma \otimes \pi \quad \forall \pi .
$$

Before we prove this implication, let us show how we can use Eq. (25) to get the result. Let $\rho_{1}, \rho_{2}, \sigma_{2}, \sigma_{2}$ be such that $\rho_{1} \prec \sigma_{1}$ and $\rho_{2} \prec \sigma_{2}$. Then, by Corollary III.4 and the weak additivity of $E_{R}^{\infty}$, we find $\rho_{1}^{\otimes 1+\epsilon} \prec \prec \sigma_{1}$ and $\rho_{2}^{\otimes 1+\epsilon} \prec \prec \sigma_{2}$, for every $\epsilon>0$. Then, applying Eq. (25) twice,

$$
\rho_{1}^{\otimes 1+\epsilon} \otimes \rho_{2}^{\otimes 1+\epsilon} \prec \sigma_{1} \otimes \rho_{2}^{\otimes 1+\epsilon} \prec \sigma_{1} \otimes \sigma_{2} .
$$

The result of the lemma follows from LemmaVI.1 and the fact that $\epsilon>0$ is arbitrary.

Let us now turn to the derivation of Eq. (25). We actually show that the negation of Eq. (25) implies the negation of Eq. (24). Indeed the former reads

NOT(25) : there is a triple $\rho, \sigma, \pi$ such that $\rho \prec \prec \sigma$ and NOT $\rho \otimes \pi \prec \sigma \otimes \pi$.

The total order established in Corollary III.4 shows that impossibility of the transformation $\rho \otimes \pi \prec$ $\sigma \otimes \pi$ is equivalent to $\sigma \otimes \pi \prec \prec \rho \otimes \pi$. Then we can rewrite Eq. (27) as

NOT(25) : there is a triple $\rho, \sigma, \pi$ such that $\rho \prec \prec \sigma$ and $\sigma \otimes \pi \prec \prec \rho \otimes \pi$.

To make the identification simpler let us make the relabeling $\rho \leftrightarrow \sigma$ in the equation above to get

NOT(25) : there is a triple $\rho, \sigma, \pi$ such that $\sigma \prec \prec \rho$ and $\rho \otimes \pi \prec \prec \sigma \otimes \pi$.

The negation of Eq. (24), in turn, is the following

NOT(24) : there is a triple $\rho, \sigma, \pi$ such that $\rho \otimes \pi \prec \sigma \otimes \pi$ and NOT $\rho \prec \sigma$.

From Corollary III.4 once more, we have that the negation of $\rho \prec \sigma$ is equivalent to $\sigma \prec \prec \rho$. Thus

NOT(24) : there is a triple $\rho, \sigma, \pi$ such that $\rho \otimes \pi \prec \sigma \otimes \pi$ and $\sigma \prec \prec \rho$.

It is now clear that Eq. (28) implies Eq. (29).

We can link such a possibility of catalysis in the bipartite case to an important open problem in entanglement theory, the full additivity of the regularized relative entropy of entanglement. In turn, the latter was shown in Ref. [50] to be equivalent to the full monotonicity under LOCC of $E_{R}^{\infty}$. 
Lemma VI.3 The regularized relative entropy of entanglement is fully additive for bipartite states, i.e. for every two states $\rho \in \mathcal{D}\left(\mathbb{C}^{d_{1}} \otimes \mathbb{C}^{d_{2}}\right)$ and $\pi \in \mathcal{D}\left(\mathbb{C}^{d_{1}^{\prime}} \otimes \mathbb{C}^{d_{2}^{\prime}}\right)$,

$$
E_{R}^{\infty}(\rho \otimes \pi)=E_{R}^{\infty}(\rho)+E_{R}^{\infty}(\pi)
$$

if, and only if, there is no catalysis for entanglement manipulation under asymptotically non-entangling maps.

Proof If Eq. (30) holds true and $\rho \otimes \pi \prec \sigma \otimes \pi$, then

$$
E_{R}^{\infty}(\rho)+E_{R}^{\infty}(\pi)=E_{R}^{\infty}(\rho \otimes \pi) \geq E_{R}^{\infty}(\sigma \otimes \pi)=E_{R}^{\infty}(\sigma)+E_{R}^{\infty}(\pi),
$$

and thus, as $E_{R}^{\infty}(\rho) \geq E_{R}^{\infty}(\sigma)$, we find from Corollary $\llbracket$. 4 that $\rho \prec \sigma$.

Conversely, assume that there is no catalysis. Then from the discussion above we find that axiom 6 holds true. For every bipartite pure state $|\psi\rangle$, the regularized relative entropy of entanglement is equal to the von Neumann entropy of the reduced density matrix $S\left(\psi_{A}\right)$. It hence follows that for every bipartite state $\rho$, there is a bipartite pure state $|\psi\rangle$ such that $E_{R}^{\infty}(\rho)=E_{R}^{\infty}(\psi)$.

Let $|\psi\rangle$ and $|\phi\rangle$ be such that $E_{R}^{\infty}(\rho)=E_{R}^{\infty}(\psi)$ and $E_{R}^{\infty}(\pi)=E_{R}^{\infty}(\phi)$. From Corollary III.4 we have $\rho \prec \psi, \pi \prec \phi$ and vice versa. Then, by axiom 6 we find that $\rho \otimes \pi \prec \psi \otimes \phi$ and $\psi \otimes \phi \prec \rho \otimes \pi$, from which we find, once more from Corollary $\amalg .4$, that $E_{R}^{\infty}(\rho \otimes \pi)=E_{R}^{\infty}(\psi \otimes \phi)$. The lemma is a consequence of the additivity of $E_{R}^{\infty}$ on two pure states (which follows from the fact that for pure states the measure is equal to the entropy of entanglement).

It is an open question if we can extend the lemma to the multipartite setting. The difficulty in this case is that we do not have a simple formula for $E_{R}^{\infty}$ of pure states and hence do not know if the measure is additive for two multipartite pure states.

Acknowledgements - We gratefully acknowledge Koenraad Audenaert, Jens Eisert, Andrzej Grudka, Michał Horodecki, Ryszard Horodecki, Shashank Virmani, Reinhard Werner, Andreas Winter, and the participants in the 2009 McGill-Bellairs workshop for many interesting discussions and useful correspondences. This work is part of the QIP-IRC supported by EPSRC (GR/S82176/0) as well as the Integrated Project Qubit Applications (QAP) supported by the IST directorate as Contract Number 015848 and was supported by the Brazilian agency Conselho Nacional de Desenvolvimento Científico e Tecnológico (CNPq), an EPSRC Postdoctoral Fellowship for Theoretical Physics and a Royal Society Wolfson Research Merit Award.

[1] H.B. Callen. Thermodynamics and an Introduction to Thermostatistics. John Wiley and Sons (1985).

[2] U.M. Maurer. Secret key agreement by public discussion from common information. IEEE Trans. Inf. Theo. 39, 733 (1998).

[3] C.H. Bennett, G. Brassard, C. Crépeau, R. Jozsa, A. Peres, and W.K. Wootters. Teleporting an unknown quantum state via dual classical and Einstein-Podolsky-Rosen channels. Phys. Rev. Lett. 70, 1895 (1993).

[4] C. Carathéodory. Untersuchungen über die Grundlagen der Thermodynamik. Math. Ann. 67, 355 (1909).

[5] R. Giles. Mathematical Foundations of Thermodynamics. Pergamon, Oxford, 1964.

[6] E.H. Lieb and J. Yngvason. The Physics and Mathematics of the Second Law of Thermodynamics. Phys. Rept. 310, 1 (1999).

[7] E.H. Lieb and J. Yngvason. A Fresh Look at Entropy and the Second Law of Thermodynamics. Phys. Today 53, 32 (2000).

[8] C.H. Bennett, H.J. Bernstein, S. Popescu, and B. Schumacher. Concentrating Partial Entanglement by Local Operations. Phys. Rev. A 53, 2046 (1996). 
[9] M. Horodecki, P. Horodecki, and R. Horodecki. Mixed-State Entanglement and Distillation: Is there a Bound Entanglement in Nature? Phys. Rev. Lett. 80, 5239 (1998).

[10] G. Vidal and J.I. Cirac. Irreversibility in asymptotic manipulations of entanglement. Phys. Rev. Lett. 86, 5803 (2001).

[11] D. Yang, M. Horodecki, R. Horodecki, and B. Synak-Radtke. Irreversibility for all bound entangled states. Phys. Rev. Lett. 95, 190501 (2005).

[12] F.G.S.L. Brandão and M.B. Plenio. A Generalization of Quantum Stein's Lemma. Commun. Math. 295, 791 (2010).

[13] F. Hiai and D. Petz. The proper formula for the relative entropy an its asymptotics in quantum probability. Comm. Math. Phys. 143, 99 (1991).

[14] T. Ogawa and H. Nagaoka. Strong Converse and Stein's Lemma in the Quantum Hypothesis Testing. IEEE Trans. Inf. Theo. 46, 2428 (2000).

[15] F.G.S.L. Brandão and M.B. Plenio. Entanglement Theory and the Second Law of Thermodynamics. Nature Physics 4, 873 (2008).

[16] M. Horodecki. Quantum entanglement: Reversible path to thermodynamics. Nature Physics 4, 833 (2008).

[17] R.F. Werner. Quantum states with Einstein-Podolsky-Rosen correlations admitting a hidden-variable model. Phys. Rev. A 40, 4277 (1989).

[18] S. Popescu and D. Rohrlich. Thermodynamics and the measure of entanglement. Phys. Rev. A 56, R3319 (1997).

[19] V. Vedral and M.B. Plenio. Entanglement Measures and Purification Procedures. Phys. Rev. A 57, 1619 (1998).

[20] M. Horodecki and R. Horodecki. Are There Basic Laws of Quantum Information Processing? Phys. Lett. A 244, 473 (1998).

[21] P. Horodecki, R. Horodecki, and M. Horodecki. Entanglement and thermodynamical analogies. Acta Phys. Slov. 48, 141 (1998).

[22] M.B. Plenio and V. Vedral. Teleportation, entanglement and thermodynamics in the quantum world. Contemp. Phys. 39, 431 (1998).

[23] E.M. Rains. Entanglement purification via separable superoperators. quant-ph/9707002

[24] C.H. Bennett, D.P. DiVincenzo, C.A. Fuchs, T. Mor, E. Rains, P.W. Shor, J.A. Smolin, and W.K. Wootters. Quantum nonlocality without entanglement. Phys. Rev. A 59, 1070 (1999).

[25] J.I. Cirac, W. Dür, B. Kraus, M. Lewenstein. Entangling operations and their implementation using a small amount of entanglement. Phys. Rev. Lett. 86, 544 (2001).

[26] S. Virmani, S.F. Huelga, and M.B. Plenio. Classical simulatability, entanglement breaking, and quantum computation thresholds. Phys. Rev. A. 71, 042328 (2005).

[27] V. Vedral, M.B. Plenio, M.A. Rippin, P.L. Knight. Quantifying Entanglement. Phys. Rev. Lett. 78, 2275 (1997).

[28] G. Vidal and R. Tarrach. Robustness of Entanglement. Phys. Rev. A 59, 141 (1999).

[29] A.W. Harrow and M.A. Nielsen. How robust is a quantum gate in the presence of noise? Phys. Rev. A 68, 012308 (2003).

[30] V. Vedral and E. Kashefi. Uniqueness of the Entanglement Measure for Bipartite Pure States and Thermodynamics. Phys. Rev. Lett. 89, 037903 (2002).

[31] F. Morikoshi, M. Franca Santos, and V. Vedral. Accessibility of physical states and non-uniqueness of entanglement measure. J. Phys. A: Math. Gen. 37, 5887 (2004).

[32] E. Rains. A semidefinite program for distillable entanglement. IEEE Trans. Inf. Theo. 472921 (2001).

[33] T. Eggeling, K.G.H. Vollbrecht, R.F. Werner, and M.M. Wolf. Distillability via protocols respecting the positivity of partial transpose. Phys. Rev. Lett. 87, 257902 (2001).

[34] K.M.R. Audenaert, M.B. Plenio, J. Eisert. The entanglement cost under operations preserving the positivity of partial transpose. Phys. Rev. Lett. 90, 027901 (2003).

[35] S. Ishizaka and M.B. Plenio. Entanglement under asymptotic positive-partial-transpose preserving operations. Phys. Rev. A 72, 042325 (2005).

[36] M. Horodecki, J. Oppenheim, and R. Horodecki. Are the laws of entanglement theory thermodynamical? Phys. Rev. Lett. 89, 240403 (2002).

[37] C.H. Bennett, P.W. Shor, J.A. Smolin, and A.V. Thapliyal. Entanglement-Assisted Classical Capacity of Noisy Quantum Channels. Phys. Rev. Lett. 83, 3081 (1999). 
[38] C.H. Bennett, P.W. Shor, J.A. Smolin, and A.V. Thapliyal. Entanglement-assisted capacity of a quantum channel and the reverse Shannon theorem. IEEE Trans. Inf. Theo. 48, 2637 (2002).

[39] D. Dieks Communication by EPR devices. Phys. Lett. A 92, 271 (1982).

[40] W.K. Wootters and W.H. Zurek. A Single Quantum Cannot be Cloned. Nature 299, 802 (1982).

[41] G. Smith, J.A. Smolin, and A. Winter. The quantum capacity with symmetric side channels. IEEE Trans. Info. Theory 54, 9, 4208-4217 (2008).

[42] G. Smith. The private classical capacity with a symmetric side channel and its application to quantum cryptography. arXiv:0705.3838.

[43] G. Smith and J. Yard. Quantum Communication With Zero-Capacity Channels. Science 321, 1812 (2008).

[44] K.G.H. Vollbrecht and R.F. Werner. Entanglement measures under symmetry. Phys. Rev. A 64, 062307 (2001).

[45] F.G.S.L. Brandão. Quantifying entanglement with witness operators. Phys. Rev. A 72, 022310 (2005).

[46] N. Datta. Max- Relative Entropy of Entanglement, alias Log Robustness. Int. J. Quant. Inf. 7, 475 (2009).

[47] M. Horodecki and P. Horodecki. Reduction criterion of separability and limits for a class of protocols of entanglement distillation. Phys. Rev. A 59, 4206 (1999).

[48] M.J. Donald and M. Horodecki. Continuity of Relative Entropy of Entanglement. Phys. Lett. A 264, 257 (1999).

[49] D. Jonathan and M.B. Plenio. Entanglement-Assisted Local Manipulation of Pure Quantum States, Phys. Rev. Lett. 83, 3566 (1999).

[50] F.G.S.L. Brandao, M. Horodecki, M.B. Plenio, S. Virmani. Remarks on the equivalence of full additivity and monotonicity for the entanglement cost. Open Sys. Inf. Dyn. 14, 333 (2007). 